\title{
The first-factor loadings of MMPI factor scales
}

\author{
ALLEN L. EDWARDS \\ University of Washington, Seattle, Washington \\ and \\ LYNNE K. EDWARDS \\ University of Minnesota, Minneapolis, Minnesota
}

\begin{abstract}
A principal-component analysis of the Minnesota Multiphasic Personality Inventory (MMPI) item pool by Johnson, Butcher, Null, and Johnson (1984) resulted in 21 factor scales. An independent analysis of the MMPI item pool by Costa, Zonderman, McCrae, and Williams (1985) resulted in 9 factor scales. In this study, we report two analyses. In the first analysis, we scored the MMPI and an Experimental Multiphasic Personality Inventory (EMPI) for the Johnson et al. 21 factor scales and a well-established marker scale for the first MMPI factor, the MMPI Social Desirability (SD) scale. In the second analysis, we scored the MMPI and the EMPI for the Costa et al. 9 factor scales and the SD scale. In both analyses, the first-factor loadings of the scales, when scored in both the MMPI and the EMPI, were highly congruent. In both analyses, the SD scale proved to be an excellent marker variable for the first factor.
\end{abstract}

The Experimental Multiphasic Personality Inventory (EMPI) was constructed primarily for research use in conjunction with the Minnesota Multiphasic Personality Inventory (MMPI). Items in the EMPI were selected from a pool of 2,824 "normal" personality items. For each of these items, the social desirability scale value (SDSV), defined as the average rating of the item on a 9-point scale ranging from extremely socially undesirable to extremely socially desirable, was known. For each of the items, the proportion of individuals answering the item true (P[T]), in self-description, was also known.

Comparable item statistics are available for the items in the MMPI. Messick and Jackson (1961) have published the SDSVs for each of the MMPI items, and Goldberg and Rorer (1963) have published the $\mathrm{P}(\mathrm{T})$ values for the MMPI items.

For each of the items in the MMPI, a corresponding item was selected from the pool of 2,824 items in such a way as to match as closely as possible the SDSV and the $P(T)$ of the MMPI item. Items were not selected on the basis of content, except insofar as item content determines the SDSV and P(T) of the item. The 550 items thus selected form the EMPI. Thus, for each item in the MMPI, there is a corresponding item in the EMPI that has approximately the same SDSV and P(T) as the MMPI item.

A socially desirable response to a personality item has been defined as a true response if the item has an SDSV greater than 5.0 or as a false response if the item has an SDSV less than 5.0. A Social Desirability (SD) scale, in turn, has been defined as a scale in which all of the trait-

Correspondence regarding this article should be addressed to Allen L. Edwards, Department of Psychology, NI-25, University of Washington, Seattle, WA 98195. keyed responses are socially desirable responses. The SD scale of the MMPI consists of 39 items, all of which are keyed for socially desirable responses.

Because the SDSVs of the items in the EMPI are matched with the SDSVs of the items in the MMPI, it follows that if the scoring key for an MMPI scale is applied to the EMPI, the result will be an EMPI "scale" that has the same proportion of items keyed for socially desirable responses as has the MMPI scale. And, because the items in the MMPI scale and the EMPI scale are matched with respect to their SDSVs, it will also be true that both scales will have approximately the same social desirability intensity index (A. L. Edwards \& Walsh, 1963). These same considerations apply to any MMPI scale that is also scored in the EMPI.

In an early study concerning the MMPI and the EMPI, both inventories were scored for 57 MMPI trait scales (A. L. Edwards, 1966). The first-factor loadings of these scales, when scored in the EMPI, correlated .98 with the first-factor loadings of the same scales when they were scored in the MMPI. In addition, the first-factor loadings of both the EMPI and the MMPI scales correlated .90 with the proportion of items in the scales keyed for socially desirable responses.

More recently, L. K. Edwards and Clark (1987) scored the EMPI for 21 MMPI trait scales. The EMPI firstfactor loadings of these scales correlated .99 with the firstfactor loadings of the same scales when they were scored in the MMPI. In this study, the first-factor loadings of both the MMPI and EMPI scales correlated .97 with the proportion of items in the scales keyed for socially desirable responses.

Many of the MMPI trait scales involved in the studies cited above were constructed by the empirical method of contrasted groups. The empirical method of scale con- 
struction, however, offers no assurance that the items included in a given scale have any homogeneity of content.

Recently, two independent principal-component analyses of the complete MMPI item pool have been reported. By using the MMPI records of a combined sample of male and female inpatients and outpatients tested at the Missouri Department of Mental Health, Johnson, Butcher, Null, and Johnson (1984) developed 21 MMPI factor scales by means of a principal-component analysis of the MMPI items. Similarly, by using the MMPI records of a combined sample of males and females referred to the Duke University Medical Center for coronary arteriography, Costa, Zonderman, McCrae, and Williams (1985) developed a set of 9 MMPI factor scales by means of a principalcomponent analysis of the MMPI item pool. In both of these studies, items were assigned to scales on the basis of their loadings on a common factor. Consequently, these factor scales should have a greater homogeneity of content than the standard scales that are ordinarily scored in the MMPI.

The present study reports on the correlations of these factor scales with the SD scale when they are scored in both the MMPI and the EMPI. We also report on the firstfactor loadings of these scales when they are scored in both the MMPI and the EMPI. The structural properties, but not the content, of these factor scales will remain the same regardless of whether they are scored in the MMPI or the EMPI.

\section{METHOD}

The MMPI sample consisted of the records of 572 male and $270 \mathrm{fe}-$ male college students. All results reported are based on the total sample of 842 students. ${ }^{1}$ The EMPI sample consisted of the records of 310 male and 307 female college students. Again, results reported are based on the combined sample of 617 students.

The SD scale and the Johnson et al. 21 factor scales were scored in both the MMPI and the EMPI. ${ }^{2}$ A principal-component analysis of the 22 scales, when scored in the MMPI, resulted in a first principal component with an eigenvalue of 4.96 and accounted for approximately $23 \%$ of the total variance. For the 22 scales, when scored in the EMPI, the first principal component had an eigenvalue of 5.66 and accounted for approximately $26 \%$ of the total variance.

When the SD scale and the nine Costa et al. scales were scored in the MMPI, ${ }^{3}$ the first principal component had an eigenvalue of 3.94 and accounted for $39 \%$ of the total variance. The first principal component of these same scales, when scored in the EMPI, had an eigenvalue of 4.50 and accounted for $45 \%$ of the total variance.

\section{RESULTS}

\section{The Johnson et al. Factor Scales}

Table 1 shows the first-factor loadings of the scales when they are scored in both the MMPI and the EMPI. Tucker's coefficient of congruence between the two sets of factor loadings is equal to .94 and indicates that the first factors of both the EMPI and the MMPI are highly congruent. ${ }^{4}$

Note also that the first factors of both the EMPI and the MMPI are well-defined. The same 10 scales have salient loadings (absolute values of .40 or higher) of the
Table 1

First-Factor Loadings of the 21 Factor Scales and the SD Scale When Scored in Both the MMPI and the EMPI

\begin{tabular}{|c|c|c|c|c|}
\hline \multirow[b]{2}{*}{ Scales } & \multicolumn{2}{|c|}{$\begin{array}{l}\text { First-Factor } \\
\text { Loadings }\end{array}$} & \multicolumn{2}{|c|}{$\begin{array}{c}\text { Correlations } \\
\text { with the SD } \\
\text { Scale }\end{array}$} \\
\hline & MMPI & EMPI & MMPI & EMPI \\
\hline 1. Neuroticism & -.84 & -.78 & -.84 & -.75 \\
\hline 2. Psychoticism & -.65 & -.75 & -.48 & -.58 \\
\hline 3. Cynicism & -.60 & -.71 & -.49 & -.58 \\
\hline 4. Denial of Somatic Problems & .57 & .33 & .42 & .34 \\
\hline 5. Social Extroversion & .25 & .36 & .29 & .27 \\
\hline 6. Stereotypic Femininity & -.14 & -.25 & -.11 & -.28 \\
\hline 7. Aggressive Hostility & -.40 & -.50 & -.29 & -.35 \\
\hline 8. Psychotic Paranoia & -.59 & -.77 & -.41 & -.64 \\
\hline 9. Depression & -.65 & -.67 & -.54 & -.52 \\
\hline 10. Delinquency & -.28 & -.43 & -.14 & -.30 \\
\hline 11. Inner Directedness & .10 & -.19 & .11 & -.08 \\
\hline 12. Assertiveness & .16 & .19 & .19 & .18 \\
\hline 13. Stereotypic Masculinity & 12 & -.13 & .14 & .03 \\
\hline 14. Neurasthenic Somatization & -.56 & -.70 & -.44 & -.53 \\
\hline 15. Phobias & -.38 & -.48 & -.35 & -.31 \\
\hline 16. Family Attachment & .47 & .57 & .36 & .51 \\
\hline 17. Well-Being & .38 & .32 & .29 & .30 \\
\hline 18. Intellectual Interests & 17 & .04 & .20 & .07 \\
\hline 19. Religious Fundamentalism & .01 & .01 & -.05 & .02 \\
\hline 20. Sexual Adjustment & .58 & .42 & .45 & .32 \\
\hline 21. Dreaming & -.27 & .21 & -.18 & .14 \\
\hline 22. Social Desirability & .87 & .87 & - & - \\
\hline
\end{tabular}

Note-The last two columns of the table show the correlation coefficients of the factor scales with the SD scale when they are scored in both the MMPI and the EMPI.

same sign on both the MMPI and the EMPI first factors. Similarly, scales with low loadings on the MMPI first factor also have low loadings on the EMPI first factor. The SD scale, which has proved to be an excellent marker scale for the first MMPI factor in studies of standard MMPI scales, has the highest absolute loading on both the MMPI and the EMPI first factors. Thus, both the MMPI and the EMPI first factors are not only welldefined, they are also similarly defined.

Table 1 also shows the correlations of the 21 factor scales with the SD scale when they are scored in the MMPI and the correlations of the 21 factor scales with the SD scale when they are scored in the EMPI. The correlation coefficients of the MMPI scales with the MMPI SD scale are linearly related to the correlation coefficients of these same scales with the SD scale when they are scored in the EMPI $(r=.94)$.

\section{The Costa et al. Factor Scales}

Table 2 shows the first-factor loadings of the scales when they are scored in both the MMPI and the EMPI. As was true with respect to the Johnson et al. factor scales, we find that the first-factor loadings of the Costa et al. factor scales and the SD scale when they are scored in the MMPI are linearly related to the first-factor loadings of these same scales when they are scored in the EMPI. Tucker's coefficient of congruence between the two sets of factor loadings is equal to .98 . Note also that the first factors of both the MMPI and the EMPI are not only well- 
Table 2

First-Factor Loadings of the Nine Factor Scales and the SD Scale When Scored in Both the MMPI and the EMPI

\begin{tabular}{|c|c|c|c|c|}
\hline \multirow[b]{2}{*}{ Scales } & \multicolumn{2}{|c|}{$\begin{array}{l}\text { First-Factor } \\
\text { Loadings }\end{array}$} & \multicolumn{2}{|c|}{$\begin{array}{c}\text { Correlations } \\
\text { with the SD } \\
\text { Scale }\end{array}$} \\
\hline & MMPI & EMPI & MMPI & EMPI \\
\hline 1. Neuroticism & -.86 & -.82 & -.79 & -.71 \\
\hline 2. Psychoticism/Infrequency & -.79 & -.84 & -.68 & -.78 \\
\hline 3. Masculinity vs. Femininity & .25 & .40 & .24 & .33 \\
\hline 4. Extraversion & .19 & .56 & .18 & .43 \\
\hline 5. Religious Orthodoxy & -.06 & -.30 & -.08 & -.20 \\
\hline 6. Somatic Complaints & -.72 & -.75 & -.61 & -.62 \\
\hline 7. Inadequacy & -.77 & -.79 & -.74 & -.70 \\
\hline 8. Cynicism & -.67 & -.74 & -.55 & -.60 \\
\hline 9. Intellectual Interests & .20 & .19 & .20 & .15 \\
\hline 10. Social Desirability & .93 & .90 & - & - \\
\hline
\end{tabular}

Note-The last two columns of the table show the correlation coefficients of the factor scales with the SD scale when they are scored in both the MMPI and the EMPI.

defined, they are also similarly defined, with the same six scales having salient loadings (absolute values of .67 or higher) on both the MMPI and the EMPI first factors. And, once again, we find that the SD scale is an excellent marker scale for the first factor.

Table 2 also shows the correlation coefficients of the nine factor scales with the SD scale when they are scored in both the MMPI and the EMPI. The correlation coefficients of the factor scales with the SD scale, when scored in the MMPI, are linearly related to these same correlation coefficients when the scales are scored in the EMPI $(r=.97)$.

\section{DISCUSSION}

It is our contention that individual differences in rates of social desirability responding, as measured by the SD scale or, for that matter, by factor scores on the first MMPI factor, is not a narrow trait confined to the kinds of items found in the MMPI but is rather a general personality trait.

Evidence that the SD scale measures a general personality trait is provided by an early study in this area by A. L. Edwards and Walker (1962). Individuals were administered the SD scale and also a set of 150 items in which the content was believed to be relevant to 15 of the needs described by Murray (1938). High (HSD) and low (LSD) scorers were selected on the basis of their scores on the SD scale. The proportion of individuals answering true (P[T]) to each of the 150 items was found separately for the HSD and the LSD groups and plotted against the SDSVs of the 150 items.

The regression line of $P(T)$ on SDSV was then calculated separately for the HSD and the LSD groups. As expected, the two regression lines had different slopes, with a steeper slope for the HSD group than for the LSD group. Thus, for items with high SDSVs, those falling to the right of the point of intersection, ${ }^{5}$ the HSD group was more likely to give true and socially desirable responses to the items than was the LSD group. For items falling to the left of the point of intersection, the HSD group was more likely to give false and socially desirable responses than was the LSD group.

The Edwards and Walker (1962) study has been replicated by A. L. Edwards and Diers (1963) and by A. L. Edwards, Gocka, and Holloway (1964) with the use of different sets of personality items. The three studies cited cover a wide range of item content and indicate that individual differences in the tendency to give socially desirable responses, as measured by the SD scale, is a general personality trait. They leave little doubt that if a personality item has a high SDSV, it is more likely to be answered true by high scorers on the SD scale than by low scorers. Similarly, if an item has a low SDSV, it is more likely to be answered false by high scorers on the SD scale than by low scorers. It is important to emphasize that this finding is not restricted to the items in the MMPI.

Thus, the relationship between the first-factor loadings of the MMPI scales when they are scored in the MMPI and when these same scales are scored in the EMPI can be accounted for by the fact that the SDSVs of the paired items in the scales are comparable. In other words, what the MMPI items and the EMPI items in a given MMPI scale have in common is not "psychopathological" content but rather content that is similar in rated social desirability. Simply because an MMPI item may have a relatively low SDSV of 2.0 and a content that is obviously relevant to "psychopathology" does not mean that a corresponding EMPI item with an SDSV of 2.0 must also necessarily have a content that is relevant to psychopathology. Items with a wide range of content may all have comparable SDSVs.

\section{REFERENCES}

Costa, P. T., Zonderman, A. B., McCrae, R. R., \& Williams, R. B., JR. (1985). Content and comprehensiveness in the MMPI: An item factor analysis in a normal adult sample. Journal of Personality \& Social Psychology, 45, 925-933.

EDWARDS, A. L. (1966). A comparison of 57 MMPI scales and 57 experimental scales matched with the MMPI scales in terms of item social desirability scale values and probabilities of endorsement. Educational \& Psychological Measurement, 26, 15-27.

Edwards, A. L., \&iers, C. J. (1963). Neutral items as a measure of acquiescence. Educational \& Psychological Measurement, 22, 501-509.

Edwards, A. L., Gocka, E. F., \& Holloway, H. (1964). The development of an MMPI acquiescence scale. Journal of Clinical Psychology, 20, 148-150.

EDWARDS, A. L., \& WALKER, J. N. (1962). Relationship between probability of item endorsement and social desirability scale value for high and low groups on Edwards' SD scale. Journal of Abnormal \& Social Psychology, 64, 458-460.

EdWARDS, A. L., WALSH, J. A. (1963). The relationship between the intensity of the social desirability keying of a scale and the correlation of the scale with Edwards' SD scale and the first factor loading of the scale. Journal of Clinical Psychology, 19, 200-203.

Edwards, L. K., \& Clark, C. L. (1987). A comparison of the first factor of the MMPI and the first factor of the EMPI: The PSD factor. Educational \& Psychological Measurement, 47, 1165-1173.

Goldberg, L. R., \& RoRer, L. G. (1963). Test-retest item statistics for original and reversed MMPI items (ORI Research Monograph No. 1). Eugene: Oregon Research Institute.

Johnson, J. H., Butcher, J. N., Null, C., \& Johnson, K. N. (1984). Replicated item level factor analysis of the full MMPI. Journal of Personality \& Social Psychology, 47, 105-114.

Messick, S., JACKSON, D. N. (1961). Desirability scale values and dispersions for MMPI items. Psychological Reports, 8, 409-414.

MurRAy, H. A. (1938). Explorations in personality. New York: Oxford University Press.

Ten Berge, J. M. F. (1986). Some relationships between descriptive comparisons of components from different studies. Multivariate Behavioral Research, 21, 29-40.

\section{NOTES}

1. It should be noted that both the Johnson et al. factor scales and the Costa et al. factor scales were developed with the use of a combined sample of males and females.

2. The SD scale had 14 items in common with the Johnson et al. Neuroticism scale. These items were not scored in the Neuroticism scale. 
3. The SD scale had 8 items in common with the Costa et al. Neuroticism scale, 11 items in common with the Psychoticism scale, 1 item in common with the Masculinity versus Femininity scale, 1 item in common with the Extraversion scale, 3 items in common with the Somatic Complaints scale, and 3 items in common with the Inadequacy scale. These items were not scored in the factor scales.

4. Ten Berge (1986) lists a number of attractive properties of the coefficient of congruence and points out that, of the various other measures of agreement between two vectors of factor loadings that have been proposed, each one lacks one or more of the attractive properties of the coefficient of congruence.

5. The point of intersection of the two regression lines varies slightly with different sets of items but, on the average, it is approximately 5.8 on the 9-point rating scale.

(Manuscript received December 3, 1990.) 